\title{
Hedgehog does the two-step
}

In flies, olfactory receptor neurons (ORNs) in the antenna and maxillary palp project to glomeruli in the antennal lobe. The spatial arrangement of different classes of ORN cell bodies roughly corresponds with that of their projections in the antennal lobe. Luo and colleagues now show that, in Drosophila melanogaster, two-stage Hedgehog $(\mathrm{HH})$ signalling contributes to this spatial patterning.

$\mathrm{HH}$ signalling occurs when $\mathrm{HH}$ binds to and inactivates the Patched (PTC) receptor. This results in disinhibition of the transmembrane protein Smoothened (SMO) and ultimately triggers the transcription of several $\mathrm{HH}$ target genes, including ptc. In D. melanogaster larvae, $\mathrm{HH}$ is produced in the posterior compartment of the antennal disc (from which the antenna and maxillary palp develop), whereas PTC staining was only visible in the anterior compartment. This suggests that $\mathrm{HH}$ diffusion across the compartment border induces ptc expression in the anterior compartment.

The differential expression of $\mathrm{HH}$ and PTC was maintained in the antenna and maxillary palp in pupae, such that PTC levels are high in some ORN classes but low in others. When the authors inactivated $\mathrm{HH}$ in the antennal disc after the differential PTC expression had been established, high-PTC classes showed much more axon mistargeting than low-PTC classes. The targeting defects in high-PTC classes could be rescued by direct (that is, without $\mathrm{HH}$ signalling) overexpression of peripheral PTC, indicating that peripheral $\mathrm{HH}$ signalling maintains high PTC expression in these classes and that this is required for their axon targeting.

$\mathrm{HH}$ protein is also expressed in the pupal brain when ORNs make targeting decisions. Following knock down of $\mathrm{HH}$ levels only in the brain, low-PTC - but not high-PTC - ORN classes showed targeting defects. Overexpression of PTC or of a PTC variant that cannot bind $\mathrm{HH}$ but does inhibit SMO in normally low-PTC ORNs caused mistargeting, indicating that low-PTC classes require $\mathrm{SMO}$ activity induced by brain-derived $\mathrm{HH}$ signalling for appropriate axon targeting.

Taken together, these findings point to a double role for $\mathrm{HH}$ signalling: peripheral $\mathrm{HH}$ signalling is required to induce and maintain PTC expression in a subset of ORN classes in the antenna and maxillary palp. The resulting differential PTC levels determine the sensitivity of different ORN classes to central $\mathrm{HH}$ signalling once they arrive at the antennal lobe, such that central $\mathrm{HH}$ signalling is required only by low-PTC classes for axon targeting.
Future research will establish whether this two-step process reflects a general mechanism for establishing spatial correspondence between peripheral neurons and their targets in the brain.

Leonie Welberg

ORIGINAL RESEARCH PAPER Chou, Y. H. et al. Patterning axon targeting of olfactory receptor neurons by coupled hedgehog signaling at two distinct steps. Cell 142, 954-966 (2010)

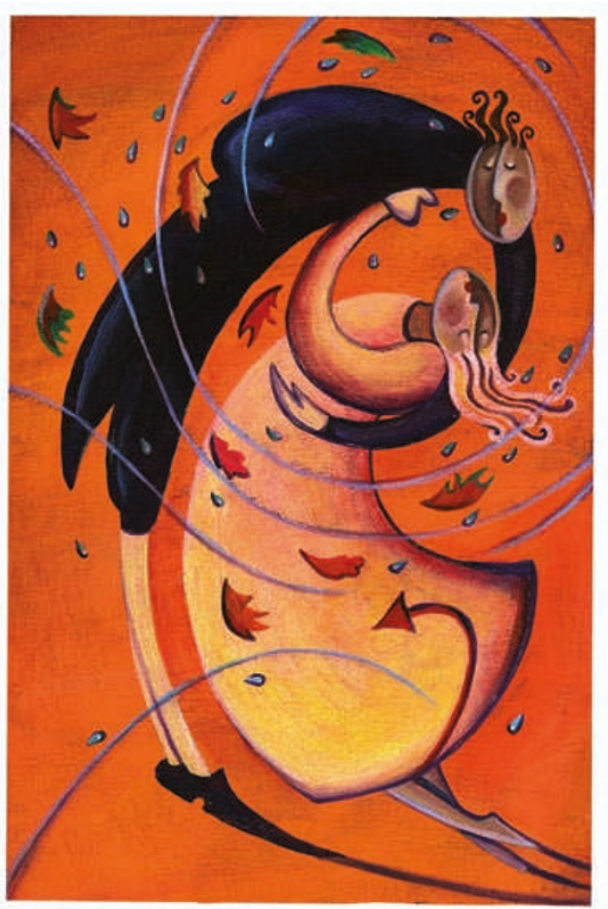

